\title{
Metal-polyaniline nanofibre composite for supercapacitor applications
}

\author{
S H KAZEMI ${ }^{\mathrm{a}, \mathrm{b}}, *$, M A KIANI $^{\mathrm{c}}$, R MOHAMADI $^{\mathrm{a}}$ and L ESKANDARIAN ${ }^{\mathrm{d}}$ \\ ${ }^{a}$ Department of Chemistry, Institute for Advanced Studies in Basic Sciences (IASBS), Zanjan 45137-66731, Iran \\ ${ }^{\mathrm{b}}$ Centre for Research in Climate Change and Global Warming (CRCC), Institute for Advanced Studies in \\ Basic Sciences (IASBS), Zanjan 45137-66731, Iran \\ ${ }^{\mathrm{c}}$ Chemistry and Chemical Engineering Research Center of Iran, P.O. Box 14335-186, Tehran, Iran \\ ${ }^{\mathrm{d}}$ Department of Textile Engineering, Amirkabir University of Technology, Hafez Ave, Tehran, Iran
}

MS received 29 January 2013; revised 20 April 2013

\begin{abstract}
The aim of the present work is to increase the electrical conductivity and specific capacitance of the polyaniline (PANi) nanofibres by introducing the metallic nanostructures. Herein, metal nanoparticleincorporated PANi nanofibres were prepared from interfacially synthesized PANi nanofibres as seeds. In the main step of aniline polymerization, the seeds were employed to produce a large amount of PANi nanofibres in the next steps. Also, metal-PANi nanofibres were chemically prepared by adding inorganic salts (nickel and copper salts) which incorporated PANi nanofibres via the self-assembly process. Increased conductivity and good electrochemical behaviour were observed for these metal-PANi nanofibres at room temperature compared with the single PANi nanofibres, which was previously reported. SEM, FT-IR and UV-Vis techniques were applied for characterization of the products. Finally, the potential application of the composites to use as electrode materials for supercapacitor was examined. Elevated specific capacitance in addition to good cycle stability was observed for the metal-PANi nanofibres. Also, electrochemical impedance spectroscopy and charge/discharge experiments show that these metal-PANi nanofibres possess the high conductivity and low charge transfer resistance, which make them suitable candidates for high-performance supercapacitors.
\end{abstract}

Keywords. Polyaniline nanofibre; nanoparticles; seeding polymerization; supercapacitor.

\section{Introduction}

Supercapacitors are defined as a class of electrochemical energy storage devices that has been attracting considerable attention in the recent years. Supercapacitors can maintain the capability to store higher energy density compared with the conventional capacitors, also deliver higher power density compared with batteries and fuel cells (Winter and Brodd 2004). Carbon materials, conductive polymers and metal oxides are the most important types of electrode materials for supercapacitors. Among these materials, intense attention has been focused on the redox pseudocapacitive materials (i.e. conducting polymers and metal oxides) (Simon and Gogotsi 2008). Recently, conducting polymers have attracted intense interests in research and applications due to their interesting chemical, electrical and optical properties, Polyaniline (PANi) is one of the most important conducting polymers because of its high conductivity, easy and economical fabrication process, very good redox properties, high stability and a wide range of applications (Huang 2003, 2004, 2006; Bhadra et al 2009; Beachley and Wen

*Author for correspondence (habibkazemi@iasbs.ac.ir)
2010, Guan et al 2010). Theoretically, specific capacitance of PANi is able to reach $2000 \mathrm{~F} \mathrm{~g}^{-1}$ (Li et al 2009). However, based on the synthetic method, morphology and the thickness of the PANi film, the specific capacitances can be varied in the range of $160-815 \mathrm{~F} \mathrm{~g}^{-1}$ (Aldissi 1993; Li et al 2012; Mu et al 2013; Tan et al 2013; Zhou et al 2013). Although in the most cases high specific capacitance of PANi electrode materials have been reported, they represent low-rate capabilities and poor cycling performance (Wang et al 2010). In the recent years, polymer composites have been utilized to overcome these limitations (Xu et al 2010; Zhang et al 2010; Han et al 2012; Su et al 2012).

Recently, nanofibre seeding polymerization was used for the synthesis of PANi nanofibres using a small amount of PANi nanofibres (Zhang et al 2004). It is concluded that seeding would make the polymerization of aniline with small amounts of nanofibres to considerably change the morphology of the resulting doped-polyaniline powder from nonfibrillar particulate to almost exclusively nanofibres. As previously reported, the formation of nanofibres takes place in nucleation and initial growth stages. Then, the nanofibres act as nucleation centres for further polymerization of other aniline monomers (secondary growth stage). In fact, the secondary growth of polyaniline 
particles leads to formation of the irregular shape of the polyaniline in micro-size scale. Therefore, it is necessary to suppress this stage. In the presence of PANi seeds, the aniline monomer would contact the seeds nearly at the same time that could speed up the induction time and force the monomers to polymerize in a certain orientation (Zhang 2004). Hence, bulk quantities of doped-PANi nanofibres were obtained via a simple route (Wnek 1988; Wang et al 2001; Mosqueda et al 2006; Thanpitcha et al 2008; Yuan et al 2010; Long et al 2011; Lu et al 2011; Safavi et al 2011). In the present work, we are introducing a simple method to synthesize PANi nanofibres with conventional polymerization of $\mathrm{PANi}$ in $\mathrm{HCl}$ by seeding approach. The synthesized nanofibres showed high conductivity and uniform and regular morphology. In addition, the effects of the metal and metal cations, including $\mathrm{Cu}$ and $\mathrm{Ni}$ cations, on the PANi morphology were examined to improve the conductivity of the PANi nanofibres. These new nanocomposites were studied as electrode materials for electrochemical supercapacitor by using cyclic voltammetry, constant current charge/discharge and a.c.-impedance techniques.

\section{Experimental}

\subsection{Materials}

Aniline was purchased from Aldrich and distilled under reduced pressure prior to each experiment. Ammonium peroxydisulfate (APS) as the oxidant, hydrochloric acid and other chemicals were purchased from Merck and Aldrich and used as-received.

\subsection{Synthesis of PANi nanofibres and metal-PANi nanofibres}

As much as $68 \mathrm{mg}$ of dissolved APS in $1 \mathrm{M} \mathrm{HCl}$ was added to a solution of aniline $(3.2 \mathrm{mmoL})$ in $\mathrm{HCl}$ rapidly, and then the mixture with total volume of $20 \mathrm{~mL}$ was sonicated for $30 \mathrm{~min}$ in an ultrasonic bath. The mixture was stirred at room temperature overnight and the resulting precipitate was centrifuged and washed with distilled water to remove excess inorganic materials. The product was desiccated under vacuum at $65^{\circ} \mathrm{C}$ for $8 \mathrm{~h}$. The resulting product, PANi nanofibres, was used as 'seeds' for the next polymerization step. In the next step, aniline is added to $10 \mathrm{~mL}$ of $1 \mathrm{M} \mathrm{HCl}$ solution containing $0.8 \mathrm{mmoL}$ APS and PANi nanfibre seeds. Finally, nickel and copper inorganic salts $\left(1 \mathrm{~g} \mathrm{CuSO}_{4} \cdot 5 \mathrm{H}_{2} \mathrm{O}\right.$ and $1.16 \mathrm{~g}$ $\left.\mathrm{Ni}\left(\mathrm{NO}_{3}\right)_{2} \cdot 6 \mathrm{H}_{2} \mathrm{O}\right)$ were added to the resultant mixture. The resulting solution was shaken for $30 \mathrm{~min}$ and stirred overnight $(100 \mathrm{rpm})$. Finally, the mixture was centrifuged, washed with distilled water and desiccated at $65^{\circ} \mathrm{C}$.

\subsection{Characterization and electrochemical studies of metal-PANi nanofibres}

The morphology of the resulting PANi was studied using a field emission scanning electron microscopy (FE-SEM, Hitachi Japan S-4160). The structure was characterized using Fourier transform infrared spectroscopy (FT-IR Bruker Vector 22). UV-Vis spectra of the synthesized PANi were determined using a Cary 100 spectrometer. Electrochemical experiments were carried out in a solution of $1 \mathrm{M} \mathrm{H}_{2} \mathrm{SO}_{4}$ in a conventional three-electrode cell with PANi-modified electrode on a disk carbon glassy electrode of $0.0314 \mathrm{~cm}^{2}$ area; $\mathrm{Pt}$ wire and $\mathrm{Ag} / \mathrm{AgCl}$ as working, counter and reference electrodes, respectively. Working electrode was prepared by mixing $2 \mathrm{mg}$ of metal-PANi nanofibres, $2 \mathrm{mg}$ of acetylene black and $1 \mathrm{mg}$ of PVDF as binder. The current collector was Ni-foam. Cyclic voltammetry (CV) and galvanostatic charge-discharge experiments were carried out by an Autolab PGSTAT 101. Galvanostatic charge/discharge tests were done in a symmetric two electrode configuration. The EIS experiments were performed over a frequency range of $100 \mathrm{kHz}-100 \mathrm{mHz}$ with a sinusoidal voltage perturbation of $10 \mathrm{mV}$ amplitude by a ZahnerZennium potentiostat-galvanostat. Experimental impedance results were fitted to suitable equivalent circuit by the complex nonlinear least squares method.

\section{Results and discussion}

\subsection{Characterization of the metal-PANi nanofibres}

Figure 1( $\mathrm{a}$ and $\mathrm{b})$ show typical FE-SEM image and EDX plot of the metal-PANi nanofibre prepared in the present work. It is found that the average diameter of nanofibre is about $50 \mathrm{~nm}$. Detailed FE-SEM analysis revealed that nanofibre lengths are in the range of $500 \mathrm{~nm}$ up to several micrometers. Also, the average size of the nanoparticles, determined by microstructure measurement software is in the range of $50-70 \mathrm{~nm}$. It should be mentioned that the FE-SEM image of the nanofibre seeds is approximately same as that of the metal-PANi nanofibre. As seen in figure 1(b), both $\mathrm{Cu}$ and $\mathrm{Ni}$ are present in the EDX plot, which is an indication of the successful incorporation of metal elements in nanocomposite structure.

Figure 2 presents the typical UV/Vis spectrum of metal-PANi nanofibres in dimethyl formamide (DMF) solution. The absorption peaks of PANi nanofibres (dissolved in DMF) were observed at about 380 and $550 \mathrm{~nm}$. These peaks are assigned to the $\pi-\pi^{*}$ transition of the benzenoid ring and the exciton absorption of the quinoid ring, respectively. It suggests that the undoped state of PANi emeraldine base (EB) is obtained from deprotonation of PANi in DMF (Zhang et al 2004, 2008; Zhang and Wang 2006; Yuan 2010). On the basis of previously 
reported works, the polymer can be converted into the deprotonated form in basic solvents, e.g. DMF. Perhaps the sharp peak appearing at about $280 \mathrm{~nm}$ may be originated from the nickel or nickel oxide nanoparticles charge transfer features, which is previously reported by Marcius et al (2012).

Figure 3(a) is a typical representation of FT-IR spectra for metal-PANi nanofibres, which was obtained in the transmission mode. It is obvious that the characteristic peaks of conducting PANi nanofibres are in good correspondence with the previously reported works (Mosqueda 2006; Long 2011). The FT-IR spectrum shows that high wavenumber bands correspond to $\mathrm{N}-\mathrm{H}$ stretching $\left(3430 \mathrm{~cm}^{-1}\right)$ and aromatic $\mathrm{C}-\mathrm{H}$ stretching vibration $\left(2960 \mathrm{~cm}^{-1}\right)$. The group $\mathrm{N}-\mathrm{Q}-\mathrm{N}$ (Q: quinoid ring)
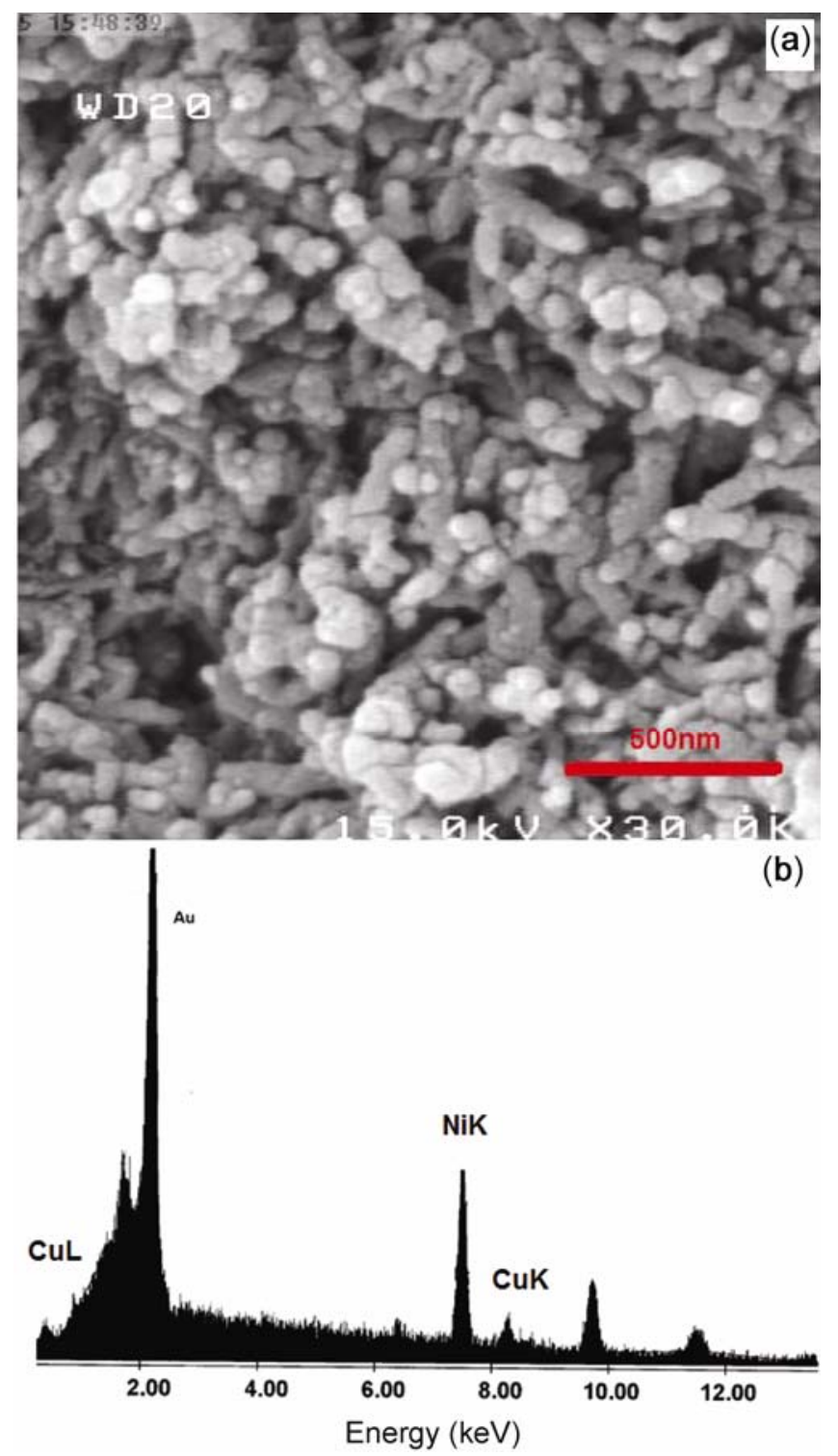

Figure 1. (a) FE-SEM image of metal-PANi nanofibres $(\mathrm{Ni} / \mathrm{Cu}-\mathrm{PANi})$ and $(\mathbf{b})$ typical EDX analysis. absorbs at $1558 \mathrm{~cm}^{-1}$ and $\mathrm{N}-\mathrm{B}-\mathrm{B}$ (B: benzenoid ring) adsorbs at $1471 \mathrm{~cm}^{-1}$. The bands at 802,1030 and $1096 \mathrm{~cm}^{-1}$ are assigned to $\mathrm{C}-\mathrm{H}$ out-of-plane and in-plane bending modes, respectively. The bands that appeared at about $1263 \mathrm{~cm}^{-1}$ might be attributed to the metal-PANi vibration modes, also $\mathrm{C}-\mathrm{H}$ stretching vibration with aromatic conjugation ( Zhang 2004, 2008; Zhang and Wang 2006). It should be mentioned that nickel oxides can be formed during the synthesis of the present metalpolyaniline nanofibre composite in addition to nickel nanoparticles; thus it is possible to observe some of the $\mathrm{NiO}$ major vibrations, which are located in the range of 390-525 $\mathrm{cm}^{-1}$ (Xing et al 2006; Ashok 2011).

\subsection{Electrochemical studies}

Cyclic voltammetry was carried out in $\mathrm{H}_{2} \mathrm{SO}_{4}$ solution $(1 \mathrm{M})$ at a scan rate of $5 \mathrm{mV} \mathrm{s}^{-1}$ (figure 4). The cyclic

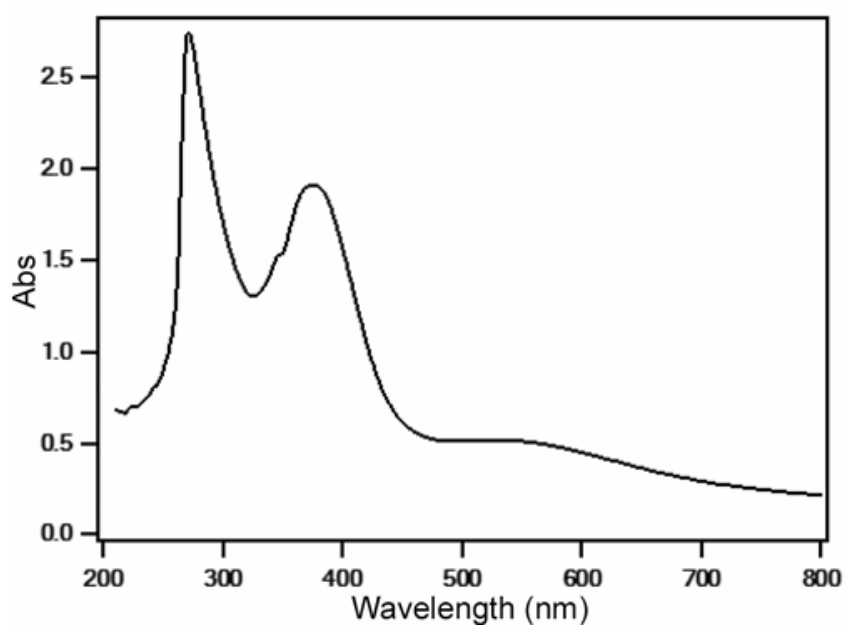

Figure 2. UV/Vis spectrum for metal-PANi nanofibres $(\mathrm{Ni} / \mathrm{Cu}-\mathrm{PANi})$ in $\mathrm{DMF}$ as solvent.

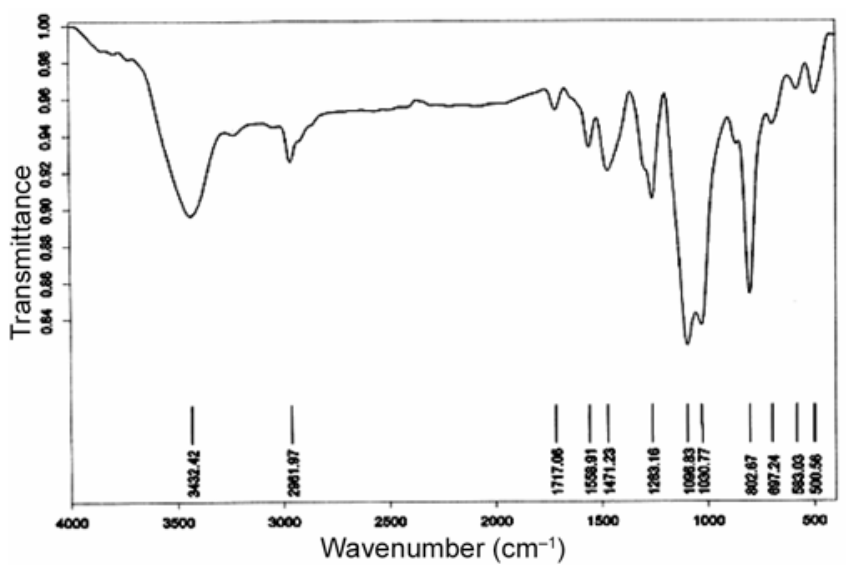

Figure 3. FT-IR spectrum for metal-PANi nanofibres $(\mathrm{Ni} / \mathrm{Cu}-\mathrm{PANi})$. 
voltammograms obtained for the metal-PANi nanofibres are in good agreement with the reported characteristics $\mathrm{CV}$ for the conducting form of PANi (Ghenaatian et al 2009; Yoon et al 2011). Successive cyclic voltammograms represent the continuous increase in peak currents, revealing that the metal-PANi nanofibre is in a conductive form (semi-oxidized form of PANi). Two couples of redox peaks appear in the $\mathrm{CV}$ curve, which are caused by the redox transitions of leucoemeraldine/polaronic emeraldine form and faradic transformation of emeraldine/ pemigraniline (Wang et al 2006). To understand the effect of metal doping into PANi nanofibres, a typical voltammogram of pure PANi nanofibres is provided in

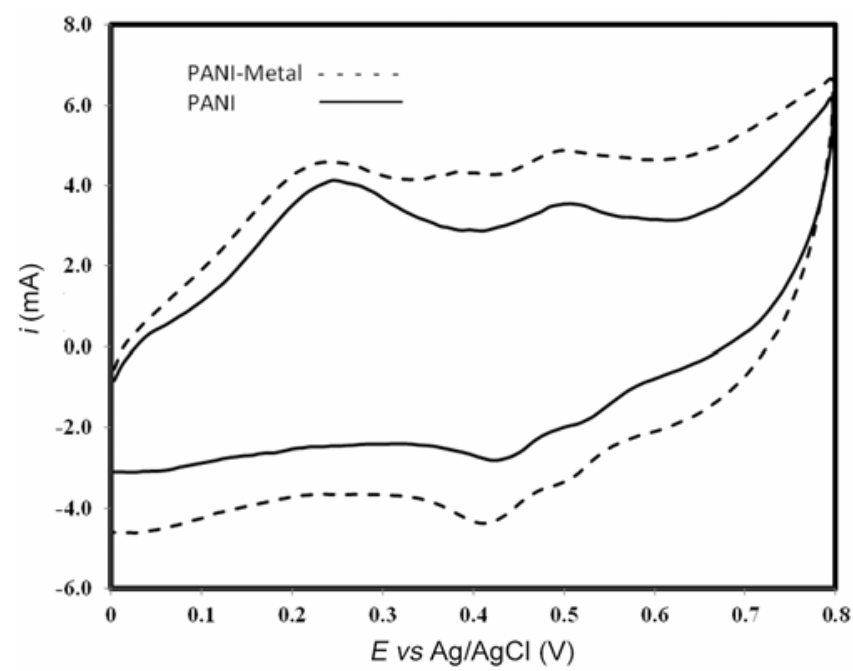

Figure 4. Typical voltammogram for metal-PANi nanofibres (dashed line) and pure PANi nanofibres (solid line) in $1 \mathrm{M}$ of $\mathrm{H}_{2} \mathrm{SO}_{4}$ at a scan rate of $5 \mathrm{~m} \mathrm{Vs}^{-1}$.

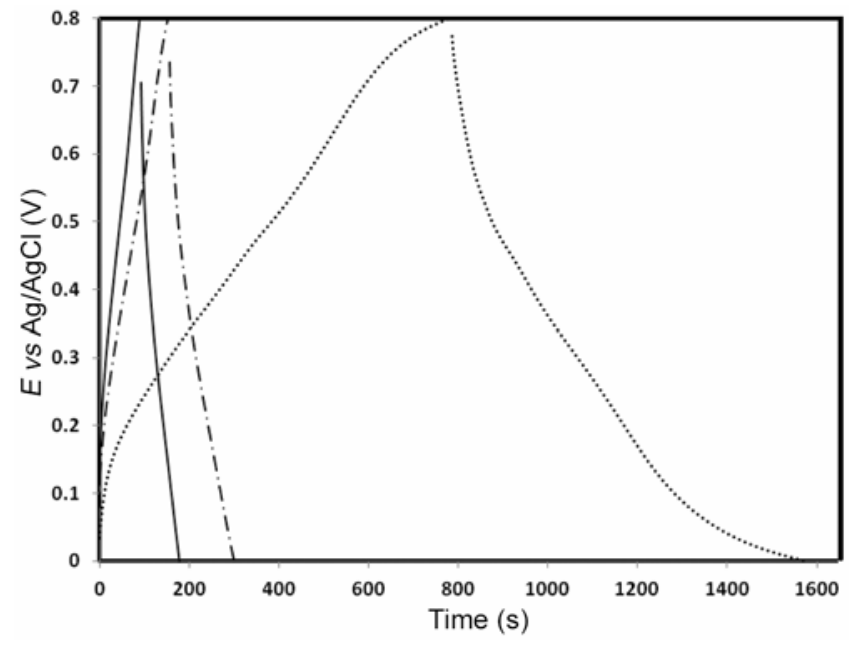

Figure 5. Galvanostatic charge/discharge plots for metalPANi nanofibres $(\mathrm{Ni} / \mathrm{Cu}-\mathrm{PANi})$ at a current density of (a) $0.86 \mathrm{~A} / \mathrm{g}$ (dotted lines), (b) $2.6 \mathrm{~A} / \mathrm{g}$ (dashed lines) and (c) $4 \cdot 3 \mathrm{~A} / \mathrm{g}$ (solid lines). figure 4. It can be concluded that the considerable enhancement in the CV currents is due to metal doping.

In order to check the charge storage capacity of newly synthesized metal-PANi nanofibres, charge/discharge experiments were performed in a three-electrode cell configuration at various current densities for metal-PANi nanofibres as electrode materials. Figure 5 shows the galvanostatic charge/discharge plots (at different current densities) of metal-PANi nanofibres. The working potential window for charge/discharge process was set as $0.8 \mathrm{~V}$ (from $0.0 \mathrm{~V}$ to $0.8 \mathrm{~V}$ ), which is consistent with the potential range of the $\mathrm{CV}$ testing (figure 5). The charging/ discharging cycling curves have a nearly symmetric shape, indicating that the composite has a good electrochemical capacitive characteristic and superior capacitive retention.

The specific capacitance, $C_{\mathrm{sp}}$, of the samples was calculated from the discharge curve of two electrode system according to the following equation:

$$
C_{\mathrm{sp}}=\frac{2 I \Delta t}{m \Delta V},
$$

where $I$ is the discharge current (A), $\Delta t$ the discharge time (s), $\Delta V$ the potential change during the discharge process and $m$ the active mass of electrodes (g). Specific capacitances of $850 \mathrm{~F} / \mathrm{g}$ at a discharge rate of $0.86 \mathrm{~A} / \mathrm{g}$ can be estimated based on the charge/discharge plots. Our results revealed that these materials are good candidates for electrode materials in supercapacitor.

The long life-cycle stability of supercapacitors is a crucial parameter for their practical application. In the present work, the cycle stability of the supercapacitor materials was carried out during successive cycles (figure 6). It is

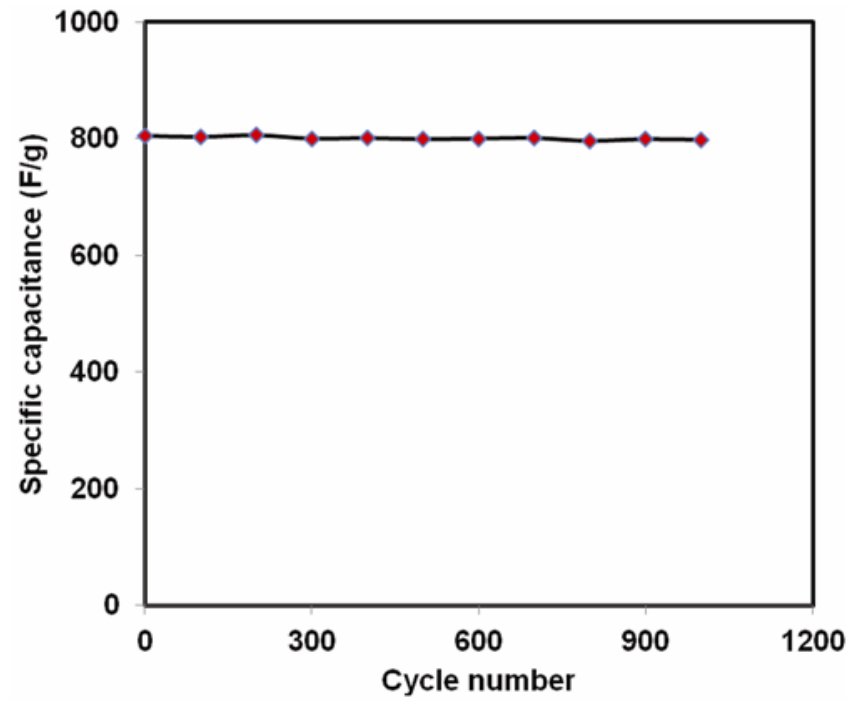

Figure 6. Cycling performance of metal-PANi nanofibres supercapacitor during the successive charge/discharge test at a current density of $1 \mathrm{~A} / \mathrm{g}$. 


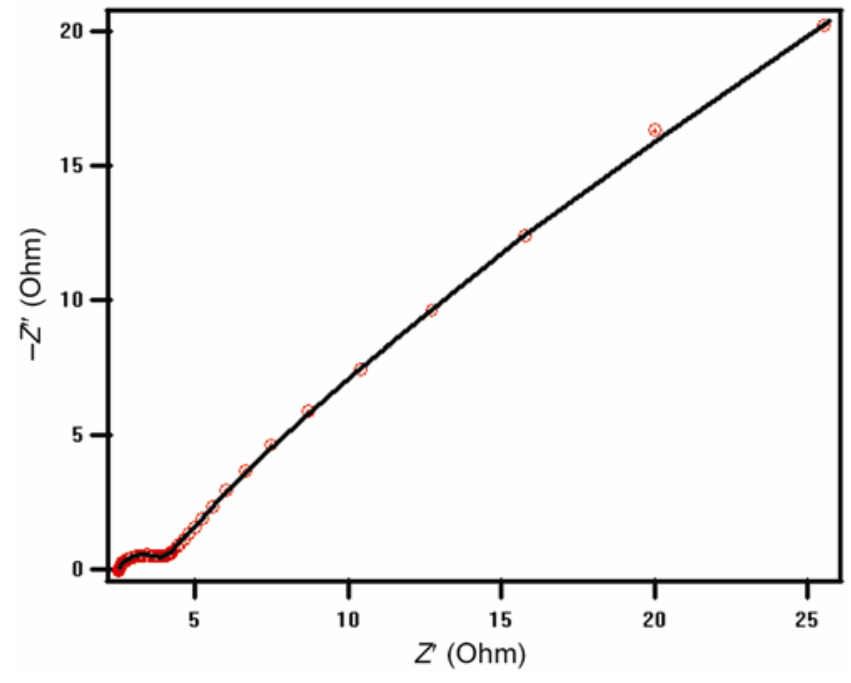

Figure 7. Nyquist diagram for metal-PANi nanofibres $(\mathrm{Ni} / \mathrm{Cu}-\mathrm{PANi})$ in $1 \mathrm{M}$ of $\mathrm{H}_{2} \mathrm{SO}_{4}$ solution at the open circuit potential. Markers are experimental data and solid line is complex nonlinear least squares fitting of experimental data.

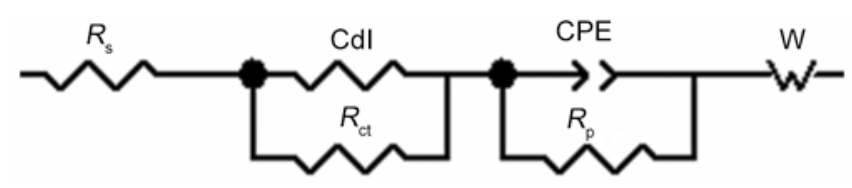

Figure 8. Equivalent circuit used in the present work to fit the EIS data (CPE and $R_{\mathrm{p}}$ are constant phase element and polymer layer resistance for metal-PANi nanofibres surface, $W$ : Warburg impedance).

observed that the specific capacitance was slightly decreased by long life cycling (the capacitance loss after 1000 charge/discharge cycles is less than $5 \%$ ). It should be noted that insignificant decrease in specific capacitance was seen after 200 successive cycles.

Electrochemical a.c. impedance analysis is an important technique to investigate the energy storage systems, especially, supercapacitors (Ghenaatian 2009; Safavi 2011). Therefore, a.c. impedance studies were done to evaluate the electrical parameters of the newly synthesized metal-PANi nanofibres. Figure 7 represents a typical Nyquist diagram of metal-PANi nanofibres-modified electrode at open circuit voltage. As seen, a small semicircle was located at high frequency region which is its diameter; charge transfer resistance in the polymeric film at the electrode surface is about $1.5 \mathrm{ohm}$. This confirms the fact that the PANi nanofibres are in the EB or semioxidized form, which show high conductivity. Impedance results were fitted with an equivalent circuit consisting of parallel combination of two RC loop circuits in series with solution resistance $\left(R_{\mathrm{s}}\right)$. The first RC loop is an explanation for the substrate surface in contact with electrolyte and the second RC loop describes the charge transfer phenomenon at the metal-PANi nanofibres $\left(R_{\mathrm{ct}}\right)$. To cover the straight part of the Nyquist diagram in the low-frequency region, Warburg impedance, $W$, was added to the second RC circuit (figure 8). The latter element describes the ion diffusion from the solution towards the surface of polymer-modified electrode. Here, the electrical parameters found by fitting are in good agreement with the results of the cyclic voltammetry and charge/ discharge experiments. More detailed impedance studies of PANi-nanofibre-based energy storage systems are in progress.

\section{Conclusions}

A simple and fast approach for preparation of metalPANi nanofibres was described in the present work. Seeding approach was used to design metal-PANi nanofibres, also spectroscopic and microscopic methods were employed to characterize them. Highly conductive nanocomposites were prepared and used as electrode material for supercapacitive purposes. Electrochemical tests are also indicated for notably high specific capacitance of metal-PANi nanofibres, which makes these materials good candidates for energy storage applications.

\section{Acknowledgement}

Financial supports of the work by the Institute for Advanced Studies in Basic Sciences are acknowledged.

\section{References}

Aldissi M 1993 Scientific affairs Division, Intrinsically conducting polymers: an emerging technology (Dordrecht: Boston, Kluwer Academic Publishers)

Ashok K 2011 Surf. Rev. Lett. 1811

Beachley V and Wen X 2010 Prog. Polym. Sci. 35868

Bhadra S, Khastgir D, Singha N K and Lee J H 2009 Prog. Polym. Sci. $\mathbf{3 4} 783$

Ghenaatian H R, Mousavi M F, Kazemi S H and Shamsipur M 2009 Synth. Met. 1591717

Guan H, Fan L-Z, Zhang H and Qu X 2010 Electrochim. Acta 56964

Han J, Li L, Fang P and Guo R 2012 J. Phys. Chem. C116 15900

Huang J X, Virji S, Weiller B H and Kaner R B 2003 J. Am. Chem. Soc. 125314

Huang J X and Kaner R B 2004 Angew. Chem. Int. Ed. 43 5817

Huang J X and Kaner R B 2006 Chem. Commun. 367

Li H, Wang J, Chu Q, Wang Z, Zhang F and Wang S 2009 J. Power Sources 190578

Li Y, Zhao K, Du X, Wang Z, Hao X, Liu S and Guan G 2012 Synthetic Metals 162107

Long Y-Z, Li M-M, Gu C, Wan M, Duvail J-L, Liu Z and Fan Z 2011 Prog. Polym. Sci. 361415 
Lu X, Zhang W, Wang C, Wen T-C and Wei Y 2011 Prog. Polym. Sci. 36671

Marciuš M, Ristić M, Ivanda M and Musić S 2012 J. Alloys Compd. $\mathbf{5 4 1} 238$

Mosqueda Y, Pérez-Cappe E, Arana J, Longo E, Ries A, Cilense M, Nascente P A P, Aranda P and Ruiz-Hitzky E 2006 J. Solid State Chem. 179308

Mu B, Liu P and Wang A 2013 Electrochim. Acta 88177

Safavi A, Kazemi, S H and Kazemi H 2011 Electrochim. Acta 569191

Simon P and Gogotsi Y 2008 Nat. Mater. 7845

Su H, Wang T, Zhang S, Song J, Mao C, Niu H, Jin B, Wu J and Tian Y 2012 Solid State Sci. 14677

Tan Q, Xu Y, Yang J, Qiu L, Chen Y and Chen X 2013 Electrochim. Acta $\mathbf{8 8} 526$

Thanpitcha T, Sirivat A, Jamieson A M and Rujiravanit R 2008 Synth. Met. 158695

Wang J, Neoh K G and Kang E T 2001 J. Colloid Interface Sci. 23978
Wang Y G, Li H Q and Xia Y Y 2006 Adv. Mater. 182619

Wang K, Huang J and Wei Z 2010 J. Phys. Chem. C114 8062

Winter M and Brodd R J 2004 Chem. Rev. 1044245

Wnek G 1988 J. Solid State Chem. 74438

Xing S, Zhao C, Jing S and Wang Z 2006 Polymer 472305

Xu J, Wang K, Zu S-Z, Han B-H and Wei Z 2010 ACS Nano 4 5019

Yoon S-B, Yoon E-H and Kim K-B 2011 J. Power Sources 196 10791

Yuan A, Wang X, Wang Y and Hu J 2010 Energy Convers. Manage. 512588

Zhang D and Wang Y 2006 Mater. Sci. Eng. B134 9

Zhang X, Goux W J and Manohar S K 2004 J. Am. Chem. Soc. 1264502

Zhang Z, Deng J, Yu L and Wan M 2008 Synth. Met. 158712

Zhang K, Zhang L L, Zhao X S and Wu J 2010 Chem. Mater. 221392

Zhou S, Zhang H, Zhao Q, Wang X, Li J and Wang F 2013 Carbon 52440 